\title{
Glomus Tumor of Uncertain Malignant Potential in the Left Upper Extremity Mimicking Leiomyosarcoma
}

\author{
Amanda L. Iavasile, Bob J. Wu \\ Bob J. Wu MD Inc, Alhambra, California, United States of America.
}

\section{Corresponding Author: \\ Amanda L. Iavasile \\ Email: aiavasile12@gmail.com}

This is an Open Access article distributed under the terms of the Creative Commons Attribution License (creativecommons.org/ licenses/by/3.0).

Received : November 21, 2016

Accepted : February 4, 2017

Published : March 20, 2017

\begin{abstract}
Background: Glomus tumors are rare tumors which usually appear in the dermis of the distal extremities in younger patients. They are categorized as benign, malignant, or of uncertain malignant potential. Given rarity of disease with a wide range of benign versus malignant behavior, the differential diagnosis is also wide and therefore may pose a diagnostic dilemma. Case Report: We present a case of glomus tumor of uncertain malignant potential in the left upper extremity of a 22 year old male, which was initially diagnosed as leiomyosarcoma upon initial excision. After re-excision achieving negative margins, a second opinion from an expert pathologist was sought and the tumor was then more accurately classified as glomus tumor of uncertain malignant potential. Close surveillance ensued with no further adjuvant treatment. Conclusion: This case highlights the difficulties in identifying and classifying glomus tumors which may significantly alter treatment plans, and therefore is considered worthy of presentation.
\end{abstract}

Keywords: Adjuvants, Dermis, Extremeties, Glomus tumor, Leiomyosarcoma.

\section{Introduction}

Glomus tumors (GTs) are uncommon benign tumors originating in the neuromyoarterial elements of the glomus body, an arteriovenous shunt specialized in thermoregulation [1]. Glomus tumors usually occur in the distal extremities of young adults and are rarely are seen in the deep soft tissue or viscera [2]. Glomus tumors have been subdivided into three groups with different prognosis: malignant glomus tumors, symplastic (benign) glomus tumors, and glomus tumors of uncertain malignant potential (GTUMP) [3]. We present a case of glomus tumor of uncertain malignant potential (GTUMP) in the left upper extremity of a young male patient which was initially diagnosed as leiomyosarcoma, highlighting the difficulties of differential diagnosis in this rare tumor with a wide continuum between benignity and malignancy.

\section{Case Report}

A 22 year old male presented with a one year history of a left dorsal forearm mass which has increased slightly in size and with occasional pain. There was no family history of any malignancy. Physical examination revealed a superficial $1.5 \mathrm{~cm}$ left dorsal forearm skin lesion which appeared flat and ecchymotic. The patient did repeatedly scratch at the lesion making its natural appearance difficult to decipher.

Excisional biopsy of the left forearm lesion was planned. The $1.5 \mathrm{~cm}$ mass was excised under local anesthesia and sedation. On histopathology spindle cell proliferation was identified with increased mitosis, extending to the deep and lateral margins [Fig.1,2]. Initial diagnosis by the pathologist was leiomyosarcoma. 
Immunohistochemistry stains were performed, and the tumor was CD34-, S100-, SMA+, and Desmin-. A repeat wide local excision was therefore planned, and patient was also concurrently referred to a tertiary sarcoma center for further workup.

Wide local excision was done one month later, showing no residual tumor or sarcoma, and all margins were clear. Further workup at sarcoma center including CT scans revealed no metastatic tumors. With the initial pathologic diagnosis in question, a second opinion was sought from an expert pathologist. In reviewing the original specimen, a new and more accurate diagnosis of glomus tumor of uncertain malignant potential (GTUMP) was made, ruling out leiomyosarcoma. Patient was then referred to an oncologist who recommended no further adjuvant chemotherapy or radiation, but did recommend long term surveillance with routine periodic physical examination.

\section{Discussion}

There are currently three categories of glomus tumors which are prognosis specific: malignant glomus tumor with metastatic potential, symplastic (benign) glomus tumor, and glomus tumor of uncertain malignant potential (GTUMP). Malignant GT with metastatic is characterized by a sub-fascial or visceral location, $>2 \mathrm{~cm}$ in size, uncharacteristic mitotic figures, $>5$ mitoses per 50 high-power fields [HPFs], and marked nuclear atypia. Symplastic GT is a benign tumor with nuclear pleomorphism and lacking mitotic activity. Lastly, GTUMP is characterized by the lack of metastatic disease, a promising prognosis, and possesses at least one feature of malignant GT such as high mitotic activity, $>2 \mathrm{~cm}$ in size, or a deep location [3]. There differential diagnosis of a glomus tumor is wide and includes carcinoid tumor, solitary fibrous tumor, leiomyoma, leiomyosarcoma, lymphoma, and peripheral primitive neuroectodermal tumor [4].

The histology of glomus tumors consist of small, round cells that reside around dilated

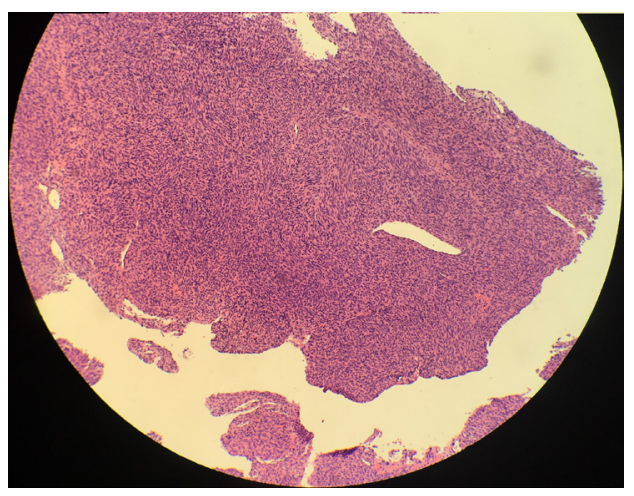

Fig.1: Glomus tumor of uncertain malignant potential in the left upper extremity (40X).

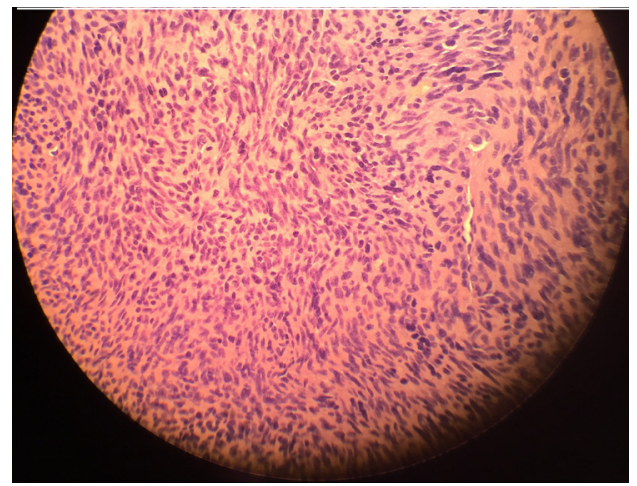

Fig.2: Glomus tumor of uncertain malignant potential in the left upper extremity (100X).

vessels. These cells also have a distinct border due to being enclosed by a basal lamina [4]. In our case, the cells were round and spindle in shape, and scattered mitosis. The presence of spindle cells as well as mitoses led to the initial diagnosis of leimyosarcoma. Further re-examination including consideration of clinical features (superficial dermal location and absence of metastasis) and immunohistochemistry was required before making the accurate diagnosis of glomus tumor of uncertain malignant potential. Typical glomus tumor immunohistochemistry demonstrates reactivity for vimentin, SMA, and caldesmon [4]. For CD34 and desmin, the staining is variable $[5,6]$. Cells surrounded by basal lamina can be outlined by immunstaining for a lamina marker, type IV collagen $[5,6]$. In this case, the neoplastic 
cells were positive for focal SMA but negative for CD34, desmin, and S100.

Treatment for malignant GTs and GTUMPs require wide surgical excision or Mohs micrographic surgery [7]. For GTUMPs, it is important to completely remove the lesion with negative margins. There is a potential for local recurrence or distant metastasis after diagnosing GTUMPs, therefore, sufficient follow-up is necessary [3]. In our case, wide local excision was achieved and CT scans demonstrated no evidence of metastasis. No adjuvant chemotherapy or radiation was recommended by an oncologist, but long term surveillance with routine periodic physical examination was proposed.

\section{Conclusion}

Glomus tumors are rare tumors which usually appear in the dermis of the distal extremities, particularly in younger patients. There is a wide continuum of benign versus malignant behavior in these tumors, at times creating difficulty in differential diagnosis. Immunohistochemistry is often helpful in diagnosis, and any glomus tumor with uncertain malignant potential should be completely excised, followed by long term surveillance.
Contributors: BJW: introduction, case report, and conclusion sections; ALI: abstract, discussion, and bibliography. ALI will act as guarantor. Both authors approved the final version of manuscript.

Funding: None; Competing interests: None stated.

\section{References}

1. Weiss SW, Goldblum JR. Perivascular tumors. In: Enzinger FM, Weiss SW, eds. Enzinger and Weiss's Soft Tissue Tumors. 5th ed. St Louis, MO: Mosby; 2008:751768.

2. Folpe AL, Fanburg-Smith JC, Miettinen M, Weiss SW. Atypical and malignant glomus tumors: analysis of 52 cases, with a proposal for the reclassification of glomus tumors. Am J Surg Pathol. 2001;25:1-12.

3. D'antonio A, Addesso M, Caleo A, Altieri R, Boscaino A. Glomus tumor of uncertain malignant potential on the forehead.Cutis. 2014;94:E13-E16

4. Wan P, Han A, Wang E, Lin X. Glomus tumor of uncertain malignant potential on the lung: a case report and review of literature. Int J Clin Exp Pathol. 2015;8:15402-15406

5. Mravic M, LaChaud G, Nguyen A, Scott MA, Dry SM, James AW. Clinical and histopathological diagnosis of glomus tumor: an institutional experience of 138 cases. Int J Surg Pathol. 2015;23:181-188.

6. Wang ZB, Yuan J, Shi HY. Features of gastric glomus tumor: a clinicopathologic, immunohistochemical and molecular retrospective study. Int J Clin Exp Pathol. 2014;7:1438-1448.

7. Cecchi R, Pavesi M, Apicella P. Malignant glomus tumor of the trunk treated with Mohs micrographic surgery [in English, German]. J Dtsch Dermatol Ges. 2011;9:391392. 